\title{
L'Entraînement doit Rester un Processus Vivant: un entretien avec Torgeir Wethal
}

\author{
Torgeir Wethal' \\ Raphaëlle Doyon"
}

'Odin Teatret - Holstebro, Danemark (in memoriam)

"Université Paris 8 - Paris, France

Torgeir Wethal est né en 1947 en Norvège et décédé en 2010 à Holstebro. Norvégien, issu de la classe moyenne, il co-fonde l'Odin Teatret en 1964, à l'âge de 17 ans après plusieurs années de pratiques théâtrales en tant qu'enfant. À partir de 1971, il monte et réalise des films sur l'Odin Teatret. Il raconte sa rencontre avec Eugenio Barba dans Erik Exe Christoffersen, The Actor's Way [1989-1993]. Cet entretien, réalisé à Florence, en Italie, le 21 mai 2005, à l'occasion d'une tournée du Rêve d'Andersen, lui rend hommage, et revient sur l'histoire et le sens du training à l'Odin Teatret.

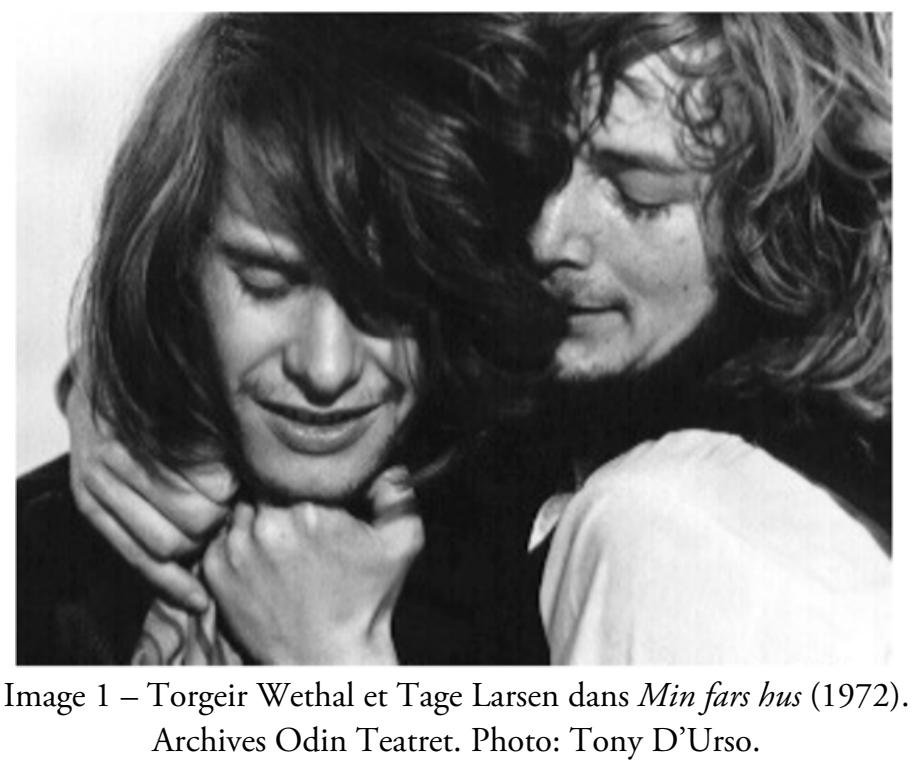

Raphaëlle Doyon - Que répondrais-tu à quelqu'un qui ne connaît pas l'Odin Teatret et te demande de décrire ce que tu fais?

Torgeir Wethal - La plupart du temps, j'essaie d'éviter de répondre à ce genre de question ou bien je m'en sors en donnant une réponse facile, succincte, qui est, en vérité, une sorte de mensonge. Cela dit, si on me pose 
la question par exemple dans la rue ou dans un bar, je répondrais que je fais du thêâtre, un type de théâtre dans lequel l'usage de la voix et du corps est plus développé que dans ce qu'on entend d'habitude par théatre. J'ajouterais que nous ne mettons pas en scène un texte écrit, mais que nous créons nos spectacles au fur et à mesure des répétitions. Je dirais quelque chose comme ça, mais ça dépend des circonstances de la discussion.

Raphaëlle Doyon - Il y a quelques années à l'Odin Teatret, tu nous avais montré, à Roberta [Carreri], Ferdinando Taviani et moi, un entretien filmé de Grotowski où il disait ceci: 'Je pense qu'il y a une façon plus rapide de devenir un vrai acteur, cela prendrait du temps, mais pas autant de temps'. J'imagine qu'il parle du temps pris par un acteur comme Ryszard Cieslak. Dans le long processus que tu as traversé pour te former, y a-t-il une chose qui selon toi n'est pas nécessaire à la formation d'un acteur? Y a-t-il un raccourci?

Torgeir Wethal - Non, il n'y a rien que je n'aurais dû faire, mais c'est, je crois, une question de génération dans cette tradition du théâtre, la mienne étant la première ou deuxième; il n'y avait personne à l'époque pour nous transmettre un modèle quotidien de travail, même si, bien sûr, nous avions les exemples des acteurs de Grotowski qui ont fortement influencé ce que je faisais et pensais, comme Ryszard [Cieslak], mais... Les êtres humains sont programmés pour imiter; c'est la manière dont on apprend depuis l'enfance, on imite et l'on devient ce que l'on apprend. Mais alors, est-ce que la façon d'apprendre importe? Bien sûr que oui, mais cette question reste ouverte pour moi. Aujourd'hui, j'utilise des tas de raccourcis dans mon enseignement aux élèves. Même les moins doués peuvent réussir à faire un équilibre sur la tête en deux jours. Il m'avait fallu deux ans. Mes élèves auront-ils perdu l'expérience que j'ai acquise pendant ces deux ans? Oui, évidemment, mais devraient-ils l'avoir? Sans doute pas pour devenir acteurs.

Raphaëlle Doyon - Alors pourquoi as-tu eu besoin, toi, de cette expérience de deux ans?

Torgeir Wethal - Parce que personne ne pouvait me l'enseigner techniquement. J'ai dû comprendre par moi-même. Bien sûr, ce n'est pas une pédagogie idiote, mais ça prend du temps. Pendant les deux années que j'ai passé à 'me cogner la tête contre les murs', j'ai appris autre chose qui continue à m'être utile aujourd'hui: je n'abandonne que très rarement une 
situation qui paraît sans espoir. C'est peut-être cela que j'ai appris et qui m’a rendu plus fort. J'ai appris à pratiquer mon obstination. Je persévère.

Mais je pense que les apprentis acteurs peuvent gagner beaucoup de temps et je ne crois pas que cela fasse d'eux des acteurs moins bons. Je crois même qu'ils peuvent devenir de meilleurs acteurs. Cela dépend aussi du sens que tu donnes au mot acteur, ou du sens que lui donnait Grotowski? Acteur pour quoi? Cela dépend de la date de l'entretien de Grotowski auquel tu fais référence.

Raphaëlle Doyon - C'était en 1976, je crois ${ }^{1}$. En novembre 2003, il y a un peu plus d'un an, j'ai interviewé Eugenio [Barba]. Il m'a raconté que quand il était avec Grotowski à Opole en 1961-63, Grotowski portait une attention particulière à Zygmunt Molik, pas à Ryszard Cieslak, à l'époque. Il m'a aussi dit qu'aux tout premiers temps de l'Odin, tu lui faisais particulièrement confiance, et qu'en raison de cela il te portait une attention autre. Tu te souviens de cela?

Torgeir Wethal - Je n'ai jamais pensé à cela en ces termes, mais c'est vrai, c'était comme cela que ça se passait. Si je me rappelle bien - mais on est toujours en train de refaire son passé, de refaire l'histoire de sa jeunesse même si j'avais tout juste dix-sept ans quand j'ai rencontré Eugenio, j'avais cette habitude, ou bien c'était un penchant naturel, de me jeter à fond dans les situations que je vivais. Ça pouvait être organiser une fête, ou créer un groupe de théâtre amateur, ou jouer un petit rôle dans des pièces de théâtre conventionnelles dans un théâtre d'Oslo. Ça pouvait être aussi le rêve de gagner de l'argent, depuis que j'avais vu des poissons remonter le fjord d'Oslo. J'avais sept ou huit ans et j'ai commencé à fabriquer des sacs en papier pour mettre les poissons pêchés. Puis, je vendais les sacs à ma famille, je faisais de la prévente. Le prix dépendait du nombre de poissons qu'ils voulaient mettre dans le sac. Je pouvais y passer des semaines. Bien sûr, il n'y avait pas de poissons dans le fjord quand je suis allé pêcher.

Quand j'ai été refusé à l'École Nationale de Théâtre d'Oslo ${ }^{2}$, ils m'ont dit que j'étais un peu trop jeune et m'ont laissé entendre que je pourrais intégrer l'école l'année d'après. Ils savaient que j'allais rester dans le milieu du théâtre. Avec l'Odin, nous avons commencé à travailler et pendant longtemps, je n'ai pas vraiment pris la décision de continuer, ou bien peutêtre que je refusais de reconnaître que j'avais pris cette décision. Le 
printemps suivant, alors que le moment était venu de passer l'audition pour essayer à nouveau d'intégrer l'école, je ne l'ai pas fait, je n'y ai même pas songé. Je suis sûr que j'étais un jeune homme très sérieux, aussi sérieux qu'on puisse l'être. À dix-sept ans, on est plus sérieux qu'à tout autre âge de la vie, du moins on pense l'être.

J'ai compris bien plus tard, même si les deux situations étaient très différentes, qu'il y avait une similitude dans la façon de se confronter à soimême dans le travail entre ce que faisait Eugenio et ce qu'avait fait mon ancien maître, professeur de théâtre. C'était un homme âgé, avec qui je travaillais pendant mon temps libre depuis l'âge de huit, neuf ans. J'ai continué à travailler avec lui jusqu'à ce que je rencontre Eugenio. Nous formions un groupe de théâtre amateur à l'école, mais c'était une activité extrascolaire. Il était Allemand et s'était échappé d'Allemagne à la fin des années 1920. Il était issu de la tradition des professeurs itinérants anthroposophes, influencés par Rudolf Steiner. La plupart d'entre eux étaient jeunes; ils voyageaient à travers l'Europe de village en village pendant la Première Guerre mondiale et enseignaient aux gens, non pas les rudiments, non pas une version vulgarisée de la musique ou de la littérature, mais ils avaient un très grand respect pour la qualité et la précision dans leur enseignement, même vis-à-vis des personnes peu instruites. Ils enseignaient la musique, la littérature, le théâtre, etc. avec les plus hauts degrés d'exigence. Bien sûr, enfant et adolescent, quand je travaillais avec lui, je ne savais pas tout cela. Cela, je l'ai lu beaucoup plus tard. Il montait avec nous des spectacles à partir de textes qu'il écrivait lui-même, souvent inspirés de contes de fées, mais pas seulement; nous montions aussi Shakespeare. Il m'avait demandé de prendre en charge le groupe avant que le cours ne commence. Je devais rassembler tout le monde afin d'être prêts à travailler quand il arrivait. Quand j'ai relu ses lettres bien des années après, environ quinze ans après avoir commencé à travailler avec Eugenio, j'ai vu les liens qu'il y avait entre l'Odin et le travail avec cet homme: le sens qu'ils donnaient aux mots, leur exigence.

\section{Torgeir fait un long silence.}

J'ai toujours plongé tête baissée dans les situations auxquelles je prenais part. Je me souviens dès les tout premiers temps, de moments sûrs, calmes, confiants dans le travail avec Eugenio qui sont devenus la base de ma 
manière de jouer, même si aucun d'entre nous, je dis nous, c'est bien cela, parce qu'aucun d'entre nous ne savait comment le travail allait évoluer. Plus tard, Grotowski et Ryszard Cieslak nous ont apporté certaines clefs, mais les fondements de notre travail se sont construits à partir de ces situations de confiance. Et il est vrai aussi qu'à l'époque où Eugenio travaillait avec Grotowski, Zygmunt [Molik] avait un rôle particulier, surtout pour son travail sur la voix ${ }^{3}$. Petit à petit, quand ils ont commencé à travailler sur Le Prince Constant, la situation a changé. L'entraînement a progressivement perdu sa fonction utilitaire de s'exercer techniquement à quelque chose pour répondre à l'exigence particulière d'un spectacle. Dans les premiers temps, les acteurs de Grotowski s'entraînaient seuls à faire des choses qu'ils devaient réaliser pratiquement le lendemain sur scène. Puis, cela a changé bien sûr ${ }^{4}$.

Raphaëlle Doyon - Avant Min fars hus, Iben [Nagel Rasmussen] a commencé à développer son propre training. Quelles ont été selon toi, les conséquences que cela a eues sur le groupe? Était-ce une proposition audacieuse? En faisant cela, elle proposait une alternative à l'entraînement préconisé, cela pouvait être interprété comme un désaccord, une opposition? Alors que bien sûr, par la suite, cet entraînement a fait le tour du monde en quelque sorte et est devenu une marque identitaire du groupe.

Torgeir Wethal - Je n'ai jamais perçu d'opposition au groupe dans le fait qu'Iben cherche un autre type d'entraînement. C'était une période où nous réagissions tous de manières différentes. J'avais l'excuse de faire des films. Au début des années 1970, je montais ou filmais quand nous n'étions pas en train de répéter. Puis, c'est devenu une espèce d'excuse pour échapper à l'entraînement. J'ai arrêté de m'entraîner plus ou moins au moment où Iben a commencé à développer son propre entraînement. Après six, sept, huit ans, cela ne m'apportait plus rien. Ensuite, le problème est que je n'ai jamais pu trouver la manière d'y revenir. Je crois que, pendant des années, cela a affaibli ma position dans le théâtre puisque je n'avais plus cette rencontre quotidienne avec le metteur en scène. Je me souviens quand nous étions en tournée, j’apportais parfois ma Moviola, ma table de montage, avec moi et je faisais du montage pendant qu'Iben travaillait dans les champs, avec Jens [Christensen] ${ }^{5}$, 19701974, par exemple quand nous étions en Suède; ils cherchaient et jouaient avec de nouvelles possibilités pour l'entraînement, j'aimais beaucoup les regarder. Et probablement, cela nous a sauvés. Le fait qu'à cette période, chacun d'entre 
nous ait pu suivre ses besoins individuels nous a probablement sauvés, nous a permis de préserver le groupe.

Là, je dois bien réfléchir parce qu'il est presque impossible de se souvenir des éléments qu'on a gardés de l'entraînement précédent avant qu'Iben n'élabore le sien. Ensuite, pendant et après Min fars hus est arrivée la génération suivante: Roberta [Carreri], Elsa [Kvamme] ${ }^{6}$, Odd [Ström] ${ }^{7}$, et plus tard Tom [Fjordefalk] ${ }^{8}$. Car évidemment, on ne passe pas d'une chose à l'autre du jour au lendemain. J'essaie de me souvenir: je sais que l'acrobatie est restée ainsi qu'une grande partie du travail de composition, certainement. En fait, il faudrait demander à Roberta ce qu'il restait de l'ancien training quand elle est arrivée, du training plastique d'autrefois et de l'entraînement physique. Je ne me souviens pas parce que je n'y participais pour ainsi dire pas. Cependant, je continuais à enseigner l'acrobatie et probablement aussi les exercices plastiques. Je me souviens qu'en 1974 à Carpignano, quand nous avons monté Le Livre des danses ${ }^{9}$, afin de pouvoir faire ces danses, j'ai dû m'entraîner pour retrouver des éléments de l'entraînement que j'avais arrêté de pratiquer depuis un bon moment déjà. J'avais donc déjà à ce moment-là complètement arrêté de m'entraîner ${ }^{10}$.

Bien sûr, quand elle sentait que c'était nécessaire, Iben avait cette manière d'être rebelle dans le travail et je ne pense pas qu'Eugenio percevait cela comme quelque chose de négatif. Ce qu'Iben a fait, de retravailler cet entraînement était une chose nécessaire sans quoi l'entraînement se serait solidifié en pierre, en une forme définie que l'on essaie d'atteindre alors que l'entraînement doit rester un processus vivant. Je me souviens que nous avions rencontré un groupe, probablement à l'époque de Ferai ${ }^{11}$, à qui l'on avait appris certains éléments du training physique, des exercices de ralenti, etc. On les a ensuite retrouvés un ou deux ans plus tard. Ils étaient capables de faire le training physique le plus difficile qui soit, des ralentis parfaits, une perfection que nous n'avions jamais vue, mais cela n'avait aucun sens. Ces exercices étaient devenus des recettes, une fin en soi. Le training n'a pas de fin en soi, si on n'en fait rien. Mais c'est quelque chose que l'on peut faire tous les jours, entrer dans la salle d'entraînement et s'entraîner, en pensant que l'on fait du théâtre. Il faut s'entraîner bien sûr, mais il faut aussi avoir quotidiennement, en parallèle, des activités théâtrales en vue d'un spectacle. Sinon, cela n'a aucun sens. 
Raphaëlle Doyon - Pourrais-tu décrire un jour de travail ordinaire de 1966-67, quand vous n'étiez pas en train de préparer un spectacle?

Torgeir Wethal - En 1966-67 nous travaillions quotidiennement à la préparation d'un spectacle! C'était la période où nous répétions et jouions Kaspariana ${ }^{12}$. Nous venions de déménager au Danemark. Au départ, nous n'avions pas de salle à nous pour travailler. Nous travaillions très tôt le matin, dans une école que l'on nous prêtait, puis de nouveau l'après-midi, quand les élèves avaient quitté les lieux. À partir du moment où nous avons eu une salle pour nous, la journée se divisait en deux temps: pendant toute la matinée, l'entraînement, à partir de sept heures; et le travail pour le spectacle l'après-midi.

Comment expliquer comment c'était à l'époque? Par exemple, j'ai été très surpris quand j'ai rencontré dans les années 1960 des groupes de théâtre qui travaillaient sur toutes sortes d'expressions physiques, parce que les acteurs s'échauffaient avant d'entrer en scène. Je n'en avais jamais entendu parler, nous ne faisions jamais ce genre de choses. Et après avoir joué, les acteurs s'étiraient. Tout cela me paraissait très étrange. Je n’ai jamais pensé au travail de l'acteur comme à un défi physique, comme c'est le cas pour le sport. Nous étions actifs, très actifs, dans le jeu, mais le processus de travail, devenu un résultat, provenait de ce que voulait mon esprit, pas de ce que pouvaient faire mes muscles.

Pendant l'entraînement, nous ne commencions probablement pas par l'acrobatie. Je ne me souviens plus très bien. Nous commencions probablement par le training plastique, travaillant successivement toutes les articulations de façon légère et ludique. Ensuite, composition pendant un bon moment, composant le corps de différentes façons. Des jambes de cigogne et un cou de cochon, inventant des figures. Nous faisions certainement environ une demi-heure de chaque, plastique puis composition, puis une demi-heure de training physique et une demi-heure d'acrobatie. Ensuite l'entraînement de la voix. Ce que nous faisions pendant l'entraînement n'était pas censé être intégré aux spectacles. Il y a eu une exception pour une courte scène de Ferai, il s'agissait d'un combat d'une minute environ qu'on avait construit à partir d'exercices d'acrobatie.

La seconde partie de la journée, nous travaillions sur un spectacle. Tous les acteurs étaient présents dans la salle pendant toute la durée des répétitions. 
De temps à autre, Eugenio continuait à me faire travailler seul sur des improvisations, et dans ce cas-là, nous n'étions que tous les deux, ou alors nous étions accompagnés par un autre acteur qui notait ce que je faisais. À partir des répétitions pour Kaspariana (1966), la plupart du temps, nous étions tous présents y compris quand c'était aux autres d'improviser, mais il arrivait encore qu'Eugenio travaille individuellement avec l'un ou l'autre des acteurs. Il me semble qu'à partir de Ferai, nous étions tous dans la salle tout le temps, même quand Eugenio conduisait un travail individuel pendant une, deux ou trois heures. Dans ce cas, les autres étaient toujours là, assis, à regarder ou réagir. Puis, ça a provoqué un énorme changement des années plus tard quand nous avons commencé à travailler individuellement ou par deux. Je ne me souviens plus très bien quand cela a commencé. Pendant la période de travail sur Oxyrhincus $^{13}$ (1985), nous étions probablement encore tous présents dans la salle de travail la plupart du temps. Aujourd'hui, chacun de nous travaille dans son coin. Aucun d'entre nous ne s'entraîne à part Kai [Bredholt] parfois, je crois.

Ou alors, si je m'entraine, c'est lorsque je travaille avec des élèves pendant les stages. Cela veut dire que ce que je fais dans une situation qui ne vise pas un spectacle en particulier, est néanmoins une préparation à des situations théâtrales futures dont je ne connais pas encore ne serait-ce que le nom ou le but. Dans ces situations, je travaille à former des idées encore branlantes, des envies, de nouvelles façons de faire. Reprendre courage, et si possible, créer des surprises. Nous ne faisons plus du tout d'équilibre sur la tête, pour ainsi dire.

\section{Notes}

1 Entretien mené par Mario Raimondo, à Holstebro en janvier 1976, pour l'émission télévisée Incontri del TG. Un'ora con Jerzy Grotowski. Transcrit dans Teatro e storia, n. 20-21, anno XIII, p. 421-429, 1998-1999.

2 Statens Teaterhøgskole (littéralement, La Grande École de Théatre de l'État) a été créée en 1953. Elle a été intégrée en 1996 à la Kunsthøgskolen i Oslo (littéralement La Grande École d'Art d'Oslo).

3 Zygmunt Molik, était "instructeur de l'action vocale: exercices respiratoires et vocaux, résonateurs, hygiène de la voix". Voir Le Théâtre-laboratoire '13 rzedów' d'Opole ou le Théâtre comme auto-pénétration collective, textes rassemblés 
par Eugenio Barba, matériaux bibliographiques annexes au mémoire Le Théâtre psychodynamique, Kraków, drukarnia Zwiazkowa, 1964.

4 À ce sujet, voir La Terre de cendres et diamants [1998], 2000, p. 60-61. Eugenio Barba explique comment est née la nécessité du training sur Akropolis en mars-avril 1962 et comment les exercices qui répondaient à des objectifs particuliers et étaient abandonnés une fois le spectacle réalisé, devinrent petit à petit autonomes vis-à-vis des spectacles.

5 Jens Christensen a été acteur à l'Odin Teatret de 1970 à 1974 . Il a joué dans Min fars hus.

6 Elsa Kvamme, actrice de l'Odin Teatret de 1973-1975; elle participe au Livre des danses (juillet 1974).

7 Odd Ström, acteur de l'Odin Teatret en 1973 et 1974; il participe au Livre des danses (juillet 1974) et à Johan Sebastian Bach.

8 Tom Fjordefalk, acteur de l'Odin Teatret de 1974 à 1984.

9 Spectacle de rue créé à Carpignano, dans le sud de l'Italie, pendant l'été 1974. 350 représentations de juillet 1974 à janvier 1980, surtout en extérieur. Le Livre des danses était un montage d'éléments du training et marque le début de la période du troc.

10 La publication de l'entretien d'Eugenio Barba en 2006 - Une vitalité radieuse (p. 27-29) - était introduite par un résumé de l'histoire interne de l'Odin Teatret dans lequel j'avais indiqué la date à laquelle Torgeir Wethal a arrêté de s'entraîner, 1971, date que l'intéressé m'avait communiquée dans cet entretien-ci de mai 2005. Eugenio Barba me fit rectifier la date de 1971 pour la remplacer par 1974. E. Barba et T. Wethal n'ont pas la même mémoire (affective?) des événements.

11 Ferai, qui relate le règne d'un jeune roi pacifique et juste après la mort d'un despote, est le troisième spectacle de l'Odin Teatret. Il se joue 220 fois entre juin 1969 et juillet 1970 .

12 Kaspariana, deuxième spectacle de l'Odin Teatret, histoire de l'éducation accélérée d'un enfant sauvage à travers une série de naissances sociales symboliques, 74 représentations entre septembre 1966 et février 1968.

13 L'Évangile selon Oxyrhincus, spectacle sur le fanatisme et le pouvoir, 214 représentations entre mars 1985 et juin 1987. 


\section{Références}

BARBA, Eugenio. Le Théâtre psychodynamique. Kraków: Drukarnia Zwiazkowa, 1964. (Mémoire tapuscrit non publié, suivi de Le Théatre-laboratoire '13 rzedów' d'Opole ou le Théâtre comme auto-pénétration collective, textes rassemblés par Eugenio Barba, matériaux bibliographiques).

BARBA, Eugenio. La Terre de Cendres et Diamants. Saussan: Entretemps, 2000. [1998].

BARBA, Eugenio. Une vitalité radieuse. Entretien réalisé par Raphaëlle Doyon. Alternatives Théâtrales, Les Liaisons singulières, n. 88, p. 27-29, $2^{\mathrm{e}}$ trimestre 2006.

CHRISTOFFERSEN, Exe. The Actor's Way. Traduit par Richard Fowler. London: Routledge, 1993. [Skuespillernes Vandring, Klim, Aarhus, 1989].

GROTOWSKI, Jerzy. Incontri del TG. Un'ora con Jerzy Grotowski. Retranscription d'une émission télévisée de Mario Raimondo tournée à Holstebro en janvier 1976. Teatro e Storia, Italia, n. 20-21, anno XIII, p. 421-429, 1998-1999.

Torgeir Wethal est né en 1947 en Norvège et décédé en 2010 à Holstebro. Norvégien, il co-fonde l'Odin Teatret en 1964, à l'âge de 17 ans.

ORCID: http://orcid.org/0000-0003-0201-3342

Raphaëlle Doyon a soutenu en 2008 à l'Université Paris 8 une thèse de doctorat intitulée L'Odin Teatret: la complémentarité des contraires. Parmi les sources de son étude figurent plusieurs entretiens qu'elle réalise dans les années 2000 avec les acteurs et actrices de l'Odin Teatret. Elle est actuellement maitresse de conférences au département Thêâtre de l'Université Paris 8. Elle publie en 2019 Les trajectoires des artistes femmes en art dramatique (http://hf-idf.org/2019/02/05/lestrajectoiresprofessionnelles-des-artistes-femmes-en-art-dramatique/).

ORCID: http://orcid.org/0000-0001-9521-4914

E-mail: doyonraphaelle@gmail.com

Cet entretien, révisé par André Mubarack, est également publié en portugais dans ce numéro.

Ce texte en libre accès est placé sous licence Creative Commons Attribution 4.0 International. Disponible sur: <http://creativecommons.org/licenses/by/4.0>. 